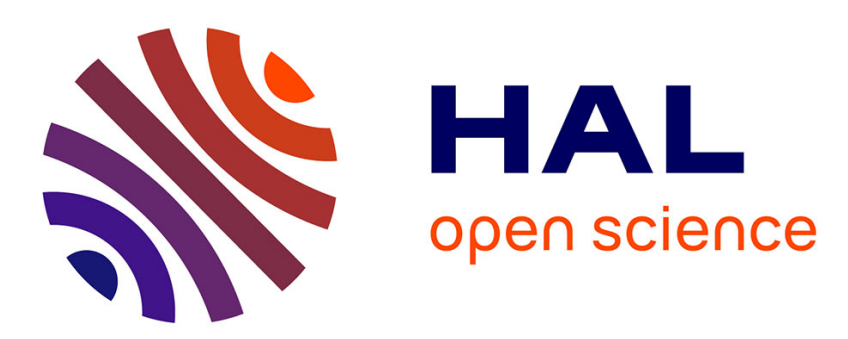

\title{
Approximate Differential Equations for Renormalization Group Functions in Models Free of Vertex Divergencies Marc Bellon
}

\section{To cite this version:}

Marc Bellon. Approximate Differential Equations for Renormalization Group Functions in Models Free of Vertex Divergencies. Nuclear Physics B, 2010, 826 [PM], pp.522-531. 10.1016/j.nuclphysb.2009.11.002 . hal-00403916v3

\section{HAL Id: hal-00403916 https://hal.science/hal-00403916v3}

Submitted on 18 Nov 2009

HAL is a multi-disciplinary open access archive for the deposit and dissemination of scientific research documents, whether they are published or not. The documents may come from teaching and research institutions in France or abroad, or from public or private research centers.
L'archive ouverte pluridisciplinaire HAL, est destinée au dépôt et à la diffusion de documents scientifiques de niveau recherche, publiés ou non, émanant des établissements d'enseignement et de recherche français ou étrangers, des laboratoires publics ou privés. 


\title{
Approximate Differential Equations for Renormalization Group Functions in Models Free of Vertex Divergencies.
}

\author{
Marc P. Bellon, ${ }^{* \dagger}$
}

\begin{abstract}
I introduce an approximation scheme that allows to deduce differential equations for the renormalization group $\beta$-function from a SchwingerDyson equation for the propagator. This approximation is proven to give the dominant asymptotic behavior of the perturbative solution. In the supersymmetric Wess-Zumino model and a $\phi_{6}^{3}$ scalar model which do not have divergent vertex functions, this simple Schwinger-Dyson equation for the propagator captures the main quantum corrections.
\end{abstract}

\section{Introduction}

New methods for efficient perturbative calculations in Quantum Field Theory are badly needed, both for practical and theoretical reasons. This work explores the possibility of approximating Schwinger-Dyson equations as differential equations on the renormalization group functions in specific cases.

Four situations will be studied and compared, based on two distinctions: the Schwinger-Dyson equation is either linear or quadratic and the theory is either a four dimensional supersymmetric model or a six dimensional scalar one. These models are characterized by the absence of divergences in vertex functions, at all orders for the Wess-Zumino model, only at one loop for the scalar model. This allows to avoid Schwinger-Dyson equations for vertex functions which would be much more difficult to study.

In the case of linear Schwinger-Dyson equations, Broadhurst and Kreimer obtained differential equations for the renormalization group functions in [1]. A generalization to the case of non-linear Schwinger-Dyson equations is the aim of this work. In a preceding work [2], we have shown how to obtain numerically high orders of the perturbative solution of a nonlinear SchwingerDyson equation, elaborating on methods proposed in 3]. These computations showed the singularities of the Borel transform of the perturbative series: the singularity on the positive axis is weaker than the one predicted by cruder approximations. However the origin of the observed asymptotic behavior of the

${ }^{*}$ UPMC Univ Paris 06, UMR 7589, LPTHE, F-75005, Paris, France

${ }^{\dagger}$ CNRS, UMR 7589, LPTHE, F-75005, Paris, France 
perturbative series was not obvious. In this work, I will introduce differential equations whose solutions are good approximations for the renormalization group function. Their iterative solutions easily show the dominant behavior of the perturbative series at high order.

Our previous paper 2] was based on two elements, which remain at the heart of the present one. A scheme for the exponentiation of the renormalization group allows to obtain the full propagator from the renormalization group function. The primitive divergence is computed with propagators to real powers, yielding a function, the Mellin transform, whose Taylor coefficients give the contribution coming from arbitrary logarithmic corrections to the propagator. Generically, this Mellin transform is a transcendent function, however a rational function can be a suitable approximation. Finally, with a rational Mellin transform, the Schwinger-Dyson equation can be converted in a differential equation for the renormalization group function. Even if these differential equations are the result of an approximation, they give the right asymptotic behavior for the perturbative series. It is then easy to determine the convergence radius of the Borel transform.

The next section will precise the models and equations we study, and recall the results in 2] which will be our starting point. Then follows the study of the Mellin transform and its singularities. Four approximate differential equations are presented with their implications. We conclude with the perspectives this work opens, in particular for Schwinger-Dyson equations including more primitives.

\section{Models and methods.}

\subsection{Schwinger-Dyson equations.}

The present study only considers massless models where renormalization is limited to the propagator. As in [2], one of these models is the supersymmetric Wess-Zumino model. The vertex functions are never divergent in this case, as can be proven to all orders in perturbation theory by superspace techniques. Another model of interest is the six-dimensional theory of a scalar complex field with the interaction:

$$
\frac{\lambda}{3 !}\left(\phi^{3}+\bar{\phi}^{3}\right)
$$

The one-loop three-point function is zero: the vertices associated to the $\phi^{3}$ interactions and those associated to its complex conjugate must alternate and this is not possible in a three point loop. Furthermore, the two-loop contribution to the three-point function is non-planar, so that it does not contribute in a large $N$ limit. It is therefore a coherent approximation to consider the Schwinger-Dyson equation associated to its unique one-loop divergence, which is a propagator correction.

The fundamental object of study of this work is therefore the simplest non- 
linear Schwinger-Dyson equation, which is graphically:

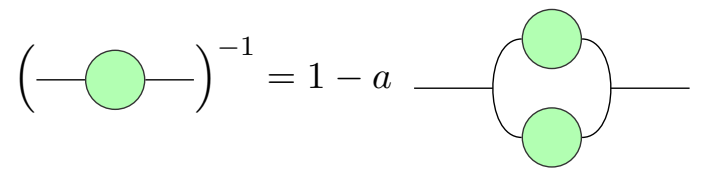

In this equation, $a$ denotes a suitable equivalent of the fine structure constant, which is equal to $\lambda^{2}$ up to some numerical constant.

The simpler linear Schwinger-Dyson equation, which has been extensively studied in [1], will also be considered as a test bed and for comparative purposes. It is graphically depicted as

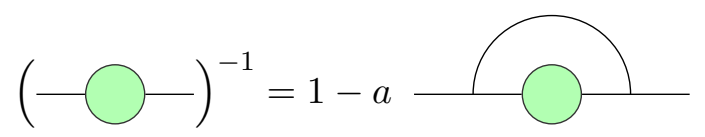

In both cases, these equations express a one-particle irreducible two-point function in term of an integral over the propagator, which is its inverse. The difficulty in providing for solutions of these Schwinger-Dyson equations stem from the need to obtain the full propagator, when only its first derivative is easily deduced. The gap is bridged by the use of the renormalization group combined with a renormalization condition taken at a fixed impulsion.

\subsection{Renormalization group.}

The two-point functions are always considered as a ratio with respect to their free counterpart. Adding a renormalization condition at a fixed exterior impulsion $p^{2}=\mu^{2}$, the two-point functions get an expansion in power of the logarithm of the impulsion $L=\log \left(p^{2} / \mu^{2}\right)$ :

$$
G(L)=1+\sum_{k} g_{k} \frac{L^{k}}{k !}
$$

I introduce here a factor $k$ ! to take $g_{k}$ as the $k^{\text {th }}$ derivative of the function, a convention which was not in either 3 or 2 but proves convenient. Now, the renormalization group relates values of the propagator at different impulsions and allows to deduce a recursive relation between the $g_{k}$. In fact, as we have seen in 2], similar recursion relations can be found for any power, positive or negative, of the propagator. The recursion has two parameters, the power $n$ of the propagator we consider and a parameter $b$ which is 2 for the linear Schwinger-Dyson equation and 3 for the non-linear one. $b$ is the power of the propagator appearing in the effective coupling constant:

$$
g_{k+1}=\gamma\left(n+b a \partial_{a}\right) g_{k} .
$$

In this equation, $\gamma$ is the anomalous dimension, the derivative of the propagator with respect to the variable $L$. I will note $\gamma_{k}$ the coefficients $g_{k}$ in the case of the propagator, with the obvious equality $\gamma_{1}=\gamma$.

The proof, detailed in [2], is based on two elements. On the one hand, the sums of the diagrams of a given order generate a sub Hopf algebra of the 
renormalization Hopf algebra [4] and this property remains true for the partial sums including only the diagrams generated by a Schwinger-Dyson equation.

On the other hand, the renormalization group is a one parameter group in the group of characters of the renormalization Hopf algebra. This fact is simpler to establish in our case than in the original works of Connes and Kreimer [5]. In the minimal subtraction scheme they used, the counterterms are not algebra homomorphism so that it is not trivial that the ratio of the counterterms at two different scales gives an algebra homomorphism. Here however, renormalization is at a fixed scale, so that the counterterm is simply the convolution inverse of the evaluation at the renormalization scale. The renormalization group is a simple consequence of the definition of the renormalized evaluation, if we know that the renormalized evaluation has a well defined limit when the regulator is removed.

$$
\begin{aligned}
\Phi_{q^{2} / p_{0}^{2}}^{R} & =\left(\Phi_{p_{0}^{2}} \circ S\right) \star \Phi_{q^{2}} \\
& =\left(\Phi_{p_{0}^{2}} \circ S\right) \star \Phi_{p^{2}} \star\left(\Phi_{p^{2}} \circ S\right) \star \Phi_{q^{2}} \\
& =\Phi_{p^{2} / p_{0}^{2}}^{R} \star \Phi_{q^{2} / p^{2}}^{R}
\end{aligned}
$$

In this equation, the convolution inverse is written using the antipode $S$ of the renormalization Hopf algebra.

The introduction of these Hopf algebra structures has for me two virtues. It allows to give a precise sense to the fact that the very same combinatorial identities which make the renormalization program work are at the base of the renormalization group. In the case we are interested in, it shows that the necessary relations hold when we do not deal with the full perturbative development, but only to the part generated by a truncated Schwinger-Dyson equation.

\subsection{Evaluation of loop integrals}

In both cases, we simply have to evaluate a single scalar loop integral. This is clear for the $\phi_{6}^{3}$ case, and was proven in [6, 2] for the supersymmetric one. Schwinger parameterization allows to obtain the following result:

$$
F(a, b)=\int \frac{d k^{D}}{\left(k^{2}\right)^{a}\left((p-k)^{2}\right)^{b}}=\pi^{\frac{D}{2}}\left(p^{2}\right)^{\frac{D}{2}-a-b} \frac{\Gamma\left(\frac{D}{2}-a\right)}{\Gamma(a)} \frac{\Gamma\left(\frac{D}{2}-b\right)}{\Gamma(b)} \frac{\Gamma\left(\frac{D}{2}-c\right)}{\Gamma(c)}
$$

with $c$ defined as $c=D-a-b$. This expression is very symmetric in $a, b$ and $c$, and this can be explained by the introduction of the two loop vacuum diagram with three propagators having the exponents $a, b$ and $c$. The choice of $c$ makes this diagram scale invariant and hence divergent. This infinite result can however be understood as a finite quantity multiplying the infinite volume of the dilatation group. If the invariance under the group of rotations and dilatations is fixed by choosing one of the impulsions, one obtains this finite coefficient. The scale can be fixed in a covariant way with $\left(p^{2}\right)^{\frac{D}{2}} \delta\left(p-p_{0}\right)$, to obtain a result independent of the impulsion which has been fixed. This enhanced symmetry is not very important in this simple one-loop case, where 
an explicit expression for the result is at hand, but afford great simplifications for higher loop cases.

At a given order, the propagator takes the form of the free propagator multiplied by a polynomial in $L=\log \left(p^{2} / \mu^{2}\right)$. It can be obtained from the action of a differential operator on $\left(p^{2}\right)^{-a}$. More precisely, we write:

$$
P\left(p^{2}\right)=\frac{1}{p^{2}}\left(1+\sum \gamma_{k} \frac{\log \left(p^{2} / \mu^{2}\right)^{k}}{k !}\right)=\left.\left(1+\sum \gamma_{k} \frac{\partial_{x}^{k}}{k !}\right) \frac{1}{\left(p^{2}\right)^{1-x}}\right|_{x=0}
$$

If we plug this value in the loop integral, we can exchange the derivation and the loop integration, so that we end up multiplying the $\gamma_{k}$ by the coefficients of the Taylor expansion around $(0,0)$ of $F(1-x, 1-y)$.

The object of interest is therefore the Taylor expansion of $F(1-x, 1-y)$. The function $F$ has poles whenever one of the $\Gamma$ function in the numerator has a negative integer as argument, i.e., for the parameters equal to $\frac{D}{2}$ or greater integers. The residues are simple polynomials. When $a$ or $b$ is a great enough integer, it corresponds to infrared divergences. For $c$ instead, the pole comes from a ultraviolet divergence, but the two are linked by conformal invariance. This link between poles of the Mellin transform and divergences of the diagram gives clues for the computation of the residues even at higher loop order, when the full Mellin transform has no closed form expression. This should allow to control the effect of higher loop corrections to the Schwinger-Dyson equation and will be the subject of future work.

\section{The differential equations}

\subsection{Linear Schwinger-Dyson equations}

In the cases with linear Schwinger-Dyson equations, differential equations for the anomalous dimension were presented in [1]. However the process through which these equations were encountered, involving explorations and computer assisted transformations of a system of partial differential equations, was not enlightening. A first explanation was presented in this same work, through the notion of propagator-coupling duality: this gives the equations we derived from the renormalization group.

The four dimensional case is really simple, without any possible variation, and is left as an exercise for the interested reader. In the $\phi_{6}^{3}$ case, equation (6) is taken with $b=1, a=1-x$ and $D=6$. The Mellin transform becomes a simple rational function. The Schwinger-Dyson equation takes the following form:

$$
\Pi(L)=1-a\left(\sum \gamma_{k} \frac{\partial_{x}^{k}}{k !}\right)\left[e^{L x}-1\right] \frac{1}{x(1+x)(2+x)(3+x)}
$$

Conformal invariance corresponds to the exchange of $a$ and $c$ and implies a symmetry for $x$ transformed to $-3-x$. The application of a suitable differential operator with respect to $L$ allows to reduce the right hand side to a constant, and the different terms of the left hand side can be obtained from $\gamma$ through the use of some version of equation (5). This derivation has been presented in Yeats' thesis [7]. 
This case will be used to test the approximation scheme I propose: let us forget the poles most removed from the origin and compensate with the first few terms of the Taylor expansion. One obtains a simpler differential equation. Instead of applying the full differential operator $\partial_{L}\left(1+\partial_{L}\right)\left(2+\partial_{L}\right)\left(3+\partial_{L}\right)$, we drop the last factors and truncate the Taylor series for $1 /(2+x)(3+x)$ to obtain a differential expression on $\gamma$ of similar order. Instead of

$$
\left(3+\gamma\left(2 a \partial_{a}-1\right)\right)\left(2+\gamma\left(2 a \partial_{a}-1\right)\right)\left(1+\gamma\left(2 a \partial_{a}-1\right)\right) \gamma=a,
$$

one obtains:

$$
\gamma+\gamma\left(2 a \partial_{a}-1\right) \gamma=\frac{a}{6}\left(1-\frac{5}{6} \gamma+\frac{19}{36} \gamma\left(2 a \partial_{a}+1\right) \gamma\right)
$$

Let us remark that the terms quadratic in $\gamma$ on the left and right hand sides of this equation differ, since in one case, one is dealing with the higher derivatives of the 1PI two-point function and in the other one, with those of its inverse, the full propagator. A comparison of numerical solutions of the two equations show that the simplified equation (10) fairs remarkably well: the two solutions are visually indiscernible up to $a=2$, the relative error is around half of a percent at $a=1$. The behaviors however diverge for larger $a$. When the exact equation (9) has a regular solution for large $a$, behaving as $a^{1 / 2}$ up to logarithmic corrections, the solution of the approximate equation (10) hits a singularity around $a=11.3684$.

The perturbative solutions can also be compared. As in [1], the coefficients of degree $n$ are multiplied by $(-1)^{n} 6^{2 n-1}$ to get natural numbers, beginning with $1,11,376$. These first coefficients are equal, since the approximation is exact up to this order. The coefficients for the approximate solution are comparable to the one of the full solution, with a ratio decreasing slowly as $n^{-\beta}$, with $\beta$ around 0.22 . This value stem from the computation of 200 coefficients. One could also study the intermediate situation where only the $\left(3+\partial_{L}\right)$ factor is dropped, but this is of limited interest.

What I wanted to show is that with the action of a suitable differential operator such that the Taylor coefficients of the Mellin transform become rapidly small, truncating the Taylor expansion to a few terms nonetheless produces sensible results. Furthermore, an equation as (10) allows to compute easily the ratio of two successive terms in the perturbative expansion, which is $n / 3$. This would be less clear from the full equation (9).

\subsection{Non-linear Schwinger-Dyson for $\phi_{6}^{3}$}

In the case of a nonlinear Schwinger-Dyson equation for the six-dimensional model, the relevant Mellin transform is:

$$
e^{L(1+x+y)} \frac{\Gamma(2+x)}{\Gamma(1-x)} \frac{\Gamma(2+y)}{\Gamma(1-y)} \frac{\Gamma(-1-x-y)}{\Gamma(4+x+y)}
$$

The poles associated to the factors $\Gamma(2+x)$ or $\Gamma(2+y)$ are farther from the origin and give contributions to the Taylor expansion which decrease at least 
as $2^{-n}$ at order $n$. The dominant contributions for the high order of the Taylor expansion come from the poles of $\Gamma(-1-x-y)$. As in the preceding case, we can differentiate with respect to $L$ in order to cancel the poles near the origin. More precisely, we need to multiply the Mellin transform by $(-1-x-y)(-x-$ $y)(1-x-y)$ which corresponds to the action of $\partial_{L}-\partial_{L}^{3}$. Using the relevant renormalization group equation for the higher derivatives of $\gamma$ and a suitable truncation of the Taylor expansion one obtains:

$$
\begin{aligned}
\gamma-\gamma\left(3 a \partial_{a}-1\right) \gamma\left(3 a \partial_{a}-1\right) \gamma & = \\
\frac{a}{6}\left(1-\frac{11}{3} \gamma\right. & \left.+\frac{a}{18}\left(49 \gamma\left(3 a \partial_{a}+1\right) \gamma+67 \gamma^{2}\right)\right)
\end{aligned}
$$

The consequences of this equation will not be detailed here, since one lacks suitable comparison points. However, it is easy to see that for large orders in $a$, the dominant term in the expansion of $\gamma$ comes from the cubic in $\gamma$ term on the left hand side. Associated to the lowest order of $\gamma$ which is $a / 6$, this proves that the ratio of successive terms is asymptotically $-n / 2$. This fixes the convergence radius of the Borel transform of the perturbative series and indicates that the main singularity is on the negative axis.

\subsection{The Wess-Zumino model, simple equation.}

The $\gamma$ function for this model was studied in 2] and a number of observations could be made on the behavior of the resulting series. The approximations we propose here will be checked against the detailed computations we made, and reciprocally, the approximations allow to prove the observed properties of the series.

Our starting point is the Mellin transform obtained in [2]:

$$
-\left(e^{L(x+y)}-1\right) \frac{\Gamma(1+x) \Gamma(1+y) \Gamma(-x-y)}{\Gamma(1-x) \Gamma(1-y) \Gamma(2+x+y)}
$$

This case presents a new problem. This Mellin transform presents a pole for $x+y=1$, but the dominant ones are the poles for $x=-1$ and symmetrically $y=-1$. This poles cannot be cancelled by derivations with respect to $L$, as the ones depending only on $x+y$. The residue of the pole for $x=-1$ is 1 for the above expression, but becomes $1-y$ or $1+x y$ due to the derivation with respect to $L$ necessary to cancel the divergence for $x=y=0$.

In a first step, let us consider the contribution coming only from $(1+x)^{-1}$. The application of the differential operator $\sum \gamma_{k} \partial_{x}^{k} / k$ ! gives the sum of the $(-1)^{k} \gamma_{k}$. Since $\gamma_{k+1}$ is deduced from $\gamma_{k}$ by the application of the operator $\gamma\left(3 a \partial_{a}+1\right)$, we obtain the formal series:

$$
\sum_{k}(-1)^{k} \gamma_{k}=\sum_{k}(-1)^{k}\left[\gamma\left(3 a \partial_{a}+1\right)\right]^{k} 1=\frac{1}{1+\gamma\left(3 a \partial_{a}+1\right)} 1 .
$$

The exact definition of the inverse does not matter, since we will be multiply by the operator to cancel it. However, since the sum of the $\gamma_{k}$ is multiplied by $a$, the operator must be permuted with the operation of multiplying by $a$. We 
therefore multiply both sides of the equation by the operator $1+\gamma\left(3 a \partial_{a}-2\right)$. Putting it all together, and adding polynomial contributions to have a result exact up to the third order, we obtain successively:

$$
\begin{aligned}
\gamma= & 2 a \gamma^{2}-a+2 a \frac{1}{1+\gamma\left(3 a \partial_{a}+1\right)} 1 \\
& {\left[1+\gamma\left(3 a \partial_{a}-2\right)\right]\left(\gamma+a-2 a \gamma^{2}\right)=2 a } \\
\gamma= & a-a \gamma-\gamma\left(3 a \partial_{a}-2\right) \gamma+2 a \gamma^{2}+2 a\left(2 a \partial_{a}+1\right) \gamma^{3}
\end{aligned}
$$

With this formula, it is easy to obtain the asymptotic growth of the coefficients in the development of $\gamma=\sum_{n}(-1)^{n-1} c_{n} a^{n}$. The term $\gamma\left(3 a \partial_{a}-2\right) \gamma$ gives the ratio of successive terms proportional to $3 n$. The next term for this ratio is easy to compute, since the term cubic in $\gamma$ and $a \gamma^{2}$ do not contribute at this level of precision. One obtains:

$$
c_{n+1} \simeq(3 n+2) c_{n}
$$

This is exactly the result obtained experimentally from the calculation in [2]. The fact that this most simple approximation has this ratio asymptotically exact up to the constant terms is at first a surprise. It nevertheless has the interesting consequence that the ratio of these approximate coefficients and the exact ones will reach a finite limit. Indeed, a product of terms which behaves asymptotically as $1+O\left(1 / n^{2}\right)$ is convergent. The comparison of the coefficients obtained from the iterative solution of equation (17) with the more precise results obtained in [2] indeed shows that their ratio, which starts at 1 for the first terms, has a limit which can be estimated to be 0.942617 after hitting a maximal deviation at 0.9250 .

In the six-dimensional theory, it was more difficult to obtain this level of precision. This can be understood from the relative sizes of the terms at a given order in $a$. The rapid growth of the coefficients of $\gamma$ makes the term of order $n$ of a product dominated by the term with one of the factors of the highest possible order. The recursive definition of $\gamma_{k}$ than shows that the coefficients of $a^{n}$ in all the $\gamma_{k}$ are of comparable size when $n$ is larger than $k$. In a product $\gamma_{1} \gamma_{k}$, the dominant contribution will come from the term of degree $n-1$ in $\gamma_{k}$, which is a factor of order $n$ smaller, and products involving $\gamma_{2}$ will make still smaller contributions.

The terms which are linear in the $\gamma_{k}$ are therefore asymptotically dominant. In the Wess-Zumino model, the pole at $x=-1$ gives the exact contribution for $y=0$ and therefore for this dominant terms, whereas in the $\phi_{6}^{3}$ model, two further poles are necessary to obtain the full contribution.

We should nevertheless expect that the terms coming from $x y /(1-x-y)$ and $x y /(1+x)$ contribute finite terms to equation (18), since they represent $n$ terms of size $1 / n$. However, $x y /(1-x-y)$ has all its Taylor coefficients positive so that there are cancellations due to the alternating signs of the $\gamma_{k}$ for a given order. The cases of $x y /(1+x)$ and $x y /(1+y)$ are more subtle. The corresponding contributions can be written as:

$$
a \gamma \frac{1}{1+\gamma\left(3 a \partial_{a}+1\right)} \gamma
$$


When they are multiplied by the operator $1+\gamma\left(3 a \partial_{a}-2\right)$, the dominant infinite series is again cancelled for this term also, and we remain with

$$
3 a\left(\gamma a \partial_{a} \gamma\right) \frac{1}{1+\gamma\left(3 a \partial_{a}+1\right)} \gamma
$$

The infinite series of terms is therefore multiplied by a term of order $a^{3}$, so that the coefficients are individually proportional to $c_{n} / n^{2}$, and their sum cannot contribute a finite term. We have thus shown that the differential equation (17) allows to predict the ratio of the successive terms of the series for $\gamma$ up to vanishing terms.

\subsection{The Wess-Zumino model: higher precision equation.}

In order to reach a higher precision on the asymptotic behavior, it is necessary to take into account the pole for $x+y=1$ and the full residue of the poles at $x$ or $y=-1$. Canceling the pole for $x+y=1$ can be achieved simply by taking derivatives with respect to $L$, the logarithm of the impulsion, to multiply the Mellin transform by $(x+y)(1-x-y)$. This however add to the complexity of the residue of the poles at $x$ and $y=-1$. The Mellin transform now reads:

$$
\begin{aligned}
\frac{\Gamma(2-x-y) \Gamma(1+x) \Gamma(1+y)}{\Gamma(2+x+y) \Gamma(1-x) \Gamma(1-y)} & \\
\frac{(1-x)(2-x)}{1+y} & +\frac{(1-y)(2-y)}{1+x}-3+3(x+y) \\
-(x+y)^{2} & +2(\zeta(3)-1) x y(x+y)+\cdots
\end{aligned}
$$

In our preceding work, we remarked that expressing the residues in terms of the product $x y$ allowed for a simpler polynomial part, but in the present case, it is better to have the different summands of the residue give similar terms. The corresponding equation for the $\gamma$ function, dropping the term proportional to $\zeta(3)-1$, is:

$$
\begin{aligned}
\gamma-\gamma(3 \nabla-1) \gamma & =-3 a+6 a \gamma-2 a \gamma(3 \nabla+2) \gamma \\
& +a(4-6 \gamma+2 \gamma(3 \nabla+1) \gamma) \frac{1}{1+\gamma(3 \nabla+1)} 1
\end{aligned}
$$

The operator $\nabla$ has been introduced as a short hand for $a \partial_{a}$ to keep down the size of the equation. The formal inverse can be removed by putting everything else on the other side:

$$
\frac{\gamma+3 a-\gamma(3 \nabla-1) \gamma-6 a \gamma+2 a \gamma(3 \nabla+2) \gamma}{4-6 \gamma+2 \gamma(3 \nabla+1) \gamma}=\frac{1}{1+\gamma(3 \nabla-2)} a
$$

In this form, the differential equation obtained by applying $1+\gamma(3 \nabla-2)$ to both sides looks rather daunting. The presence of a quotient reintroduces the necessity of series inversion that we avoided by a clever use of the renormalization group equations. Otherwise, the expansion of the derivative of the quotient, followed by a multiplication by the square of the denominator in (23) gives a polynomial equation, but with numerous terms. 
Let us remark that in any case, the derivatives with respect to $a$ get multiplied by $a \gamma$ : the total number of possible terms of a given degree in $\gamma$ is therefore limited. It is possible that the combinatorial methods of the operad of algebras with derivation introduced by Jean-Louis Loday [8] is useful to stitch together similar terms. The complexity of the obtained equation raises the question whether a systematic improvement of such approximations by the addition of the contribution of other poles of the Mellin transform is practical.

The factor $a \gamma$ coming with each derivatives has a double consequence. On one side, it ensures that perturbatively, higher derivative terms are subdominant, but this also makes the differential equation highly singular in the vicinity of $a=0$ : proving the non-perturbative existence of the solution is not straightforward.

\section{Conclusion.}

In the present paper, I have shown how to deduce from Schwinger-Dyson equations simple differential equations for the renormalization group functions. They readily give the asymptotic behavior of the perturbative series and in particular the convergence radius of the Borel transform. Through the inclusion of the contributions of more poles of the Mellin transform, it should be possible to obtain systematic improvements of the solution.

Differential equations for renormalization group functions had been proposed in recent years. In her thesis [7], Karen Yeats proposed a way to linearize the nonlinear Schwinger-Dyson equations and obtain simple differential equations for the renormalization group functions. The proposition has been applied both to QED [9] and to QCD [10]. However, the transformed Schwinger-Dyson equation is indeed linear, but with an infinite number of terms and an unknown function appears in the differential equation, with a very complex recursive definition. It is therefore not clear if in a perturbative solution, the contribution from the non-linear differential term dominates the contribution of the unknown function.

Up to now, we only considered simple Schwinger-Dyson equations, with a one-loop correction to the propagator. However, the full Schwinger-Dyson equation includes higher order terms, and we would like to know how these additional contributions modify the properties of the renormalization group functions. The difficulty a priori with such terms is the great number of propagators, each coming with its own variable, and therefore the rapid growth of the number of terms with a given number of derivatives of the Mellin transform. However the leading contributions in the Taylor expansion of the multivariable Mellin transforms correspond to its poles, which can be related to the divergences of the diagram. Indeed, with every propagator coming with a variable exponent, all subdiagrams become divergent for some choice of the exponents. A rôle should be find for the core Hopf algebra introduced in [11, 12] to organize these divergences. The poles have a simple structure, because they only depend on the sum of the Mellin variables of a given subgraph. The highly nonlinear character of such Schwinger-Dyson equations should not be a hindrance to their 
successful use. In particular, at least in a large $N$ limit where the number of primitive divergences does not grow too fast, it could be possible to show that these additional terms do not change the leading asymptotic behavior of the perturbative series.

Another desirable extension is to deal with vertex renormalization. However the vertices depend a priori on different energy scales and the full vertex is not entirely defined by its renormalization group dependence. There are also overlapping divergences, which mean that it is not possible to simply replace the sum of a vertex and its counterterm by a renormalized vertex. We must also choose the renormalization point for the vertex. In QED, the Ward identities are simpler for the vertex with a zero impulse photon, but this is not a suitable choice in a massless theory.

Whatever the successes we encounter in these improvements, this work has already delivered. It has shown how to combine Schwinger-Dyson equations and renormalization group to control the asymptotic behavior of the perturbative series for exactly renormalizable quantum field theories which are not so artificial than the ones studied in [1, 6]. This is a remarkable result, since the simple recursions developed here subsume huge number of individual graphs with their hierarchy of counterterms, with important cancellations between contributions of differing signs.

Acknowledgments: I wish to express my special thanks to Olivier Babelon who suggested to look for global properties of the Mellin transform in order to understand the asymptotic properties of its local expansion which puzzled us in our preceding work. The presentation of our previous work in seminars helped me to clarify the concepts: thanks to all who invited me and specially to the organizers of a workshop in Cargèse.

\section{References}

[1] D. J. Broadhurst and D. Kreimer. Exact solutions of Dyson-Schwinger equations for iterated one-loop integrals and propagator-coupling duality. Nucl. Phys., B 600:403-422, 2001, arXiv:hep-th/0012146.

[2] Marc Bellon and Fidel Schaposnik. Renormalization group functions for the Wess-Zumino model: up to 200 loops through Hopf algebras. Nucl. Phys. B, 800:517-526, 2008, arXiv:0801.0727v2 [hep-th].

[3] Dirk Kreimer and Karen Yeats. An etude in non-linear DysonSchwinger equations. Nucl. Phys. Proc. Suppl., 160:116-121, 2006, arXiv: hep-th/0605096.

[4] Walter D. van Suijlekom. Multiplicative renormalization and Hopf algebras. 2007, arXiv:0707.0555 [hep-th]

[5] Alain Connes and Dirk Kreimer. Renormalization in quantum field theory and the Riemann-Hilbert problem. II: The beta-function, diffeomorphisms 
and the renormalization group. Commun. Math. Phys., 216:215-241, 2001, arXiv:hep-th/0003188

[6] Marc Bellon, Gustavo Lozano, and Fidel Schaposnik. Higher loop renormalization of a supersymmetric field theory. Physics Letters B, 650:293297, 2007, arXiv:hep-th/0703185.

[7] Karen Amanda Yeats. Growth estimates for Dyson-Schwinger equations. $\mathrm{PhD}$ thesis, Boston University, 2008, arXiv:0810.2249 [math-ph].

[8] Jean-Louis Loday. On the operad of associative algebras with derivation. 2009, arXiv:0906.4730v1 [math.RA].

[9] Guillaume van Baalen, Dirk Kreimer, David Uminsky, and Karen Yeats. The QED beta-function from global solutions to Dyson-Schwinger equations. Ann. Phys., 324:205-219, 2009, arXiv:0805.0826 [hep-th].

[10] Guillaume van Baalen, Dirk Kreimer, David Uminsky, and Karen Yeats. The QCD beta-function from global solutions to Dyson-Schwinger equations. 2009, arXiv:0906.1754 [hep-th].

[11] Dirk Kreimer. The core Hopf algebra. 2009, arXiv:0902.1223 [hep-th].

[12] Dirk Kreimer and Walter D. van Suijlekom. Recursive relations in the core Hopf algebra. 2009, arXiv:0903.2849 [hep-th]. 